\title{
Application of Sentinel Node Biopsy to Non-oral Head and Neck Cancer
}

${ }^{1}$ Beejal Patel, ${ }^{2}$ Clare Schilling

\begin{abstract}
Cervical lymph node status is a significant prognostic factor for all patients with head and neck cancer. There is still ongoing deliberation on the extent of surgical therapy to offer patients, particularly those who have a clinically negative (cNO) neck. Currently, preoperative examination and investigation [routinely ultrasound, computed tomography (CT), and magnetic resonance imaging (MRI)] are utilized for treatment planning and the extent of surgery based on evidence of metastatic spread or perceived risk of occult metastasis.

It has been shown that that sentinel node biopsy (SNB) is a reliable staging test to detect occult metastases in early oral carcinoma, and its routine use has been advocated in the UK by the National Institute of Health and Clinical Excellence (NICE). Sentinel node biopsy can be used in most solid tumors that spread via lymphatics but its application to extraoral head and neck malignancies (other than primary skin tumors) has not been widely reported. In this article, we review the potential application of SNB in new areas of the head and neck.
\end{abstract}

Keywords: Laryngeal cancer, Metastasis, Neck dissection, Salivary cancer, Sentinel node, Thyroid cancer.

How to cite this article: Patel B, Schilling C. Application of Sentinel Node Biopsy to Non-oral Head and Neck Cancer. Int J Head Neck Surg 2018;11(2):71-77.

Source of support: Nil

Conflict of interest: None

\section{INTRODUCTION}

Almost one-third of the body's lymph nodes are found in the head and neck region. ${ }^{1}$ Locoregional metastases to these lymph nodes reduce survival by up to $50 \%{ }^{2}$ not only for patients affected by oral cancer but also thyroid, laryngeal, and salivary malignancies.

The SNB, initially a novel concept, was accepted into routine clinical practice following the work of Morton et al. ${ }^{3}$ in relation to malignant melanoma. The SNB was

\footnotetext{
${ }^{1}$ Core Surgical Trainee, ${ }^{2}$ Consultant

${ }^{1}$ St George's Hospital, Tooting, London, UK

${ }^{2}$ Department of Oral and Maxillofacial Surgeon, Head and Neck Academic Unit, University College Hospital, London, UK

Corresponding Author: Clare Schilling, Consultant, Department of Oral and Maxillofacial Surgeon, Head and Neck Academic Unit, University College Hospital, London, UK, e-mail: Clare. Schilling@nhs.net
}

subsequently evaluated and applied to staging of breast, colonic, urogenital, lung, and head and neck cancers.

Sentinel node biopsy works on the principle that primary tumor sites are lymphatically drained in a sequential manner, the first node or group of nodes to drain a site are known as the sentinel node(s) (SN). Identification, harvesting, and detailed pathological examination of the $\mathrm{SN}$ allow accurate detection of micrometastatic deposits (negative predictive value $94 \%^{4}$ ) and the decision for further lymph node dissection is based upon $\mathrm{SN}$ status. The standard applied is that if the $\mathrm{SN}$ are free from disease, then more distal disease is unlikely, thus in head and neck cancer, completion neck dissection is judged unnecessary following a negative SNB.

\section{TECHNIQUE AND DEVELOPMENT IN ORAL CANCER}

The standard technique of SNB involves a preoperative peritumoral injection of ${ }^{99 \mathrm{~m}}$ Tc-labeled colloid tracer often together with an intraoperative tumoral injection of blue dye or fluorescent tracer (indocyanine green) to enhance visual detection of SNs. ${ }^{5-8}$ Preoperative lymphoscintigraphy is performed within 24 hours prior to surgery and the position of identified SNs marked externally. During surgery, $\mathrm{SN}$ are identified by gamma probe detection of radioactivity and these "hot" or blue/fluorescent nodes are harvested for intensive pathological examination. Each SN undergoes serial step sectioning at $150 \mu \mathrm{m}$ intervals followed by immunohistochemical staining, allowing identification of micrometastatic deposits and viable isolated tumor cells.

A multicenter European trial (The Sentinel European Node Trial-SENT) was conducted (2005-2010) in which 415 patients with cT1/2N0 oral squamous cell carcinoma underwent SNB as staging followed by neck dissection only if positive. The 3-year results showed overall survival of 88 with $23 \%$ of patients upstaged by the test. The SNB had a sensitivity of $86 \%$, and a false-negative rate (FNR) of $14 \%{ }^{4}$ The SNB was recognized as a safe and reliable procedure associated with minimal shortterm morbidity (seroma, hematoma, local infection, and lymphedema).

In 2016, the UK's NICE performed cost-effectiveness analysis of SNB in oral cancer, recommending that it should be offered to all patients with a clinically 
N0 neck who do not require simultaneous microvascular reconstruction. ${ }^{9}$

There is potential to apply SNB to nonoral cancer patients with clinically N0 necks in an effort to ensure that they can be individually managed according to their tumor site. The evidence for this is further discussed below.

\section{SALIVARY CARCINOMA}

Salivary gland malignancy represents $0.3 \%$ of all cancer types. Because of the diversity and scarcity of salivary tumors, there is a deficit of high-level evidence regarding treatment, a fact recognized by recently published UK management guidelines. ${ }^{10}$ The rate of occult metastases in salivary malignancy is $10-30 \%{ }^{11-13}$ and in many cases, the metastatic nodes are located outside the traditional neck dissection fields. Up to $75 \%$ of positive nodes are intra- or periparotid ${ }^{11}$ and there is a recognized tendency to "skip-metastases" in levels III and IV without involvement of more proximal nodes. ${ }^{13}$

Despite Gould et al.'s ${ }^{14}$ report of SNB in salivary gland disease, there has been little advance in the topic over the last 50 years. His series of 28 patients with parotid tumors had the "angular node" subjected to frozen section analysis in order to decide if radical neck dissection was necessary. There were major limitations to his approach: Gould did not use any tracer to map the lymphatic flow but assumed that the SN would be the same in each case, and on final histopathology, 20 of the 28 cases were benign. In total, three of the malignant cases had positive SNs. Despite these flaws, his approach served as the foundation for the development of SNB in other areas of the body, but use in salivary malignancy stalled.

In 2006, a case series of six patients with parotid carcinoma was reported. ${ }^{15}$ The authors used ${ }^{99 \mathrm{~m}} \mathrm{Tc}$-labeled nanocolloid (50 MBq) injected at eight sites around the tumor. A SN was identified on static lymphoscintigraphy within 10 minutes of injection in all patients. The study group underwent SNB and concomitant levels II-IV or V neck dissection depending upon the location of the SN. Positive SNs were found in two patients and in one, there was a false-negative result. In this case, the false-negative result was attributed to an intraglandular metastatic node disrupting drainage. Lymphoscintigraphy images from this study show that the entire gland was hot, presumably due to the large number of peritumoral injections given. This combined with the poor anatomical detail gleaned from static lymphoscintigraphy would certainly preclude the identification of intra- and periglandular nodes, thus reducing the sensitivity of the technique significantly.

More recently, two case reports looked at modern techniques in salivary SNB. Schilling et al. ${ }^{16}$ used navigation surgery in parotid malignancy (Fig. 1) and Moreno et al. ${ }^{17}$

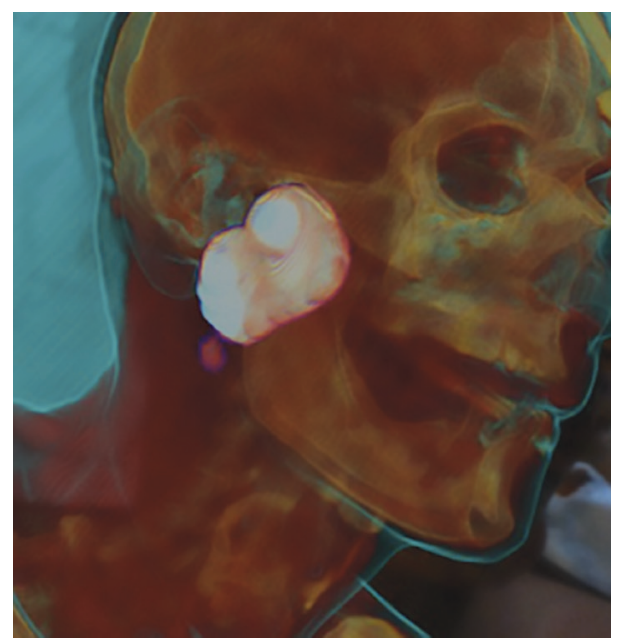

Fig. 1: Three-dimensional single photon emission CT (SPECT) projected onto patient at time of surgery demonstrating right parotid gland sentinel node identification

in adenocarcinoma of a minor salivary gland, where negative SNB resulted in conservative surgical management and avoided neck dissection without affecting outcome.

This limited evidence indicates that SNB can be applied to salivary neoplasms, but multicenter long-term trials are required to evaluate the true $\mathrm{SN}$ detection rates (which may vary depending on tumor site and epithelial origin) with identification of FNR by concurrent neck dissection or longer-term follow-up with clinical and radiological surveillance. The foreseen difficulty in evaluating this group of patients is that often postoperative radiotherapy (RT) is recommended for features of the primary tumor and the rate of nodal relapse due to false-negative SNB result may be underestimated.

\section{THYROID CARCINOMA}

Thyroid cancer is the most common type of endocrine malignancy, and constitutes $1.7 \%$ of all malignancies worldwide. The incidence of thyroid cancer has increased over the last three decades, but cure rate for differentiated thyroid cancer (DTC) remains high with an 80-90\% 10 -year survival rate, even in patients with regional metastatic disease.

Occult lymph node metastasis is thought to occur in $20-50 \%$ of DTC; ${ }^{18}$ however, the significance of this spread is debatable. Most patients will receive postoperative radioiodine, which ablates both residual thyroid tissue and metastatic disease. This offers excellent tumor control, but there is a small subset of patients who relapse. Criteria for these "high-risk" patients include male gender, age over 45 years, tumor over $4 \mathrm{~cm}$, and extracapsular or extrathyroid disease. Elective central neck dissection (clearance of levels VI and VII) is recommended in highrisk patients, although the procedure is associated with an increased risk of recurrent laryngeal nerve damage 
and hypoparathyroidism. ${ }^{19,20}$ On the opposite end of the spectrum, there are clinical trials in progress looking at whether patients with low-risk disease can be spared iodine treatment. ${ }^{21}$

There is lack of consensus about the extent of lymph node sampling required to stage both high- and low-risk patients, to which SNB may offer a solution.

Two meta-analysis reviews have looked at the use of SNB in thyroid carcinoma staging. The first analyzed 14 studies with a pool of 457 patients. A key conclusion drawn was that the type of tracer used ( ${ }^{99 \mathrm{~m}} \mathrm{Tc}-$ Colloid vs Blue dye) played an important role in SN detection rate and overall, the use ${ }^{99 \mathrm{~m}} \mathrm{Tc}-$ Colloid yielded a $13 \%$ higher detection rate. ${ }^{22}$ This review did not consider SN detection rates among studies, or how the presence of a positive SN could predict likely further nodal disease or represent skip metastases. It also failed to consider complications associated with the SNB procedure and was unable to comment on the longer term clinical implications of staging using SNB (such as recurrence), but described the practicality of the technique - which was demonstrated as feasible. A second meta-analysis by Balasubramanian and Harrison ${ }^{23}$ included 24 cohort studies evaluating the efficacy of sentinel lymph node biopsy (SLNB) in papillary thyroid cancers. This analysis yielded an overall combined $\mathrm{SN}$ detection rate of $86 \%$; however, of note, the majority of included studies used blue dye alone for detection which was by this time already shown to be less efficacious at detecting SLN. Analyzed data also acknowledged that with the use of SNB, central END could have been avoided in $57 \%$ of cN0 patients. ${ }^{23}$ In addition, $23 \%$ of $\mathrm{cN} 0$ patients were found to have a positive $\mathrm{SN}$ in the lateral neck compartment and in $15 \%$ of these patients, this was the only identified $\mathrm{SN}^{23}$ further raising two further clinical considerations regarding whether the extent of prophylactic elective neck dissections in this group of patients is enough and the question of when lateral neck compartment dissection should be performed.

Data on FNR for detection of metastases in lymph nodes varied greatly between studies, mostly reflecting the variation in nodal examination methods (serial section, immunohistochemistry, or frozen section) with combination of serial section and immunohistochemistry showing the highest sensitivity range from 84 to $100 \%{ }^{24,25}$

A third review ${ }^{26}$ concluded that SNB additionally identifies positive nodes outside of the central compartment allowing for more rigorous individual surgical and extension of lymphadenectomy if required. ${ }^{26}$ In this scenario, SNB could help stratify patients with local metastatic disease who would benefit from Iodine ablation therapy vs avoidance of iodine ablation in low-risk or neck-negative patient groups. Heterogeneity between the majority of available studies unfortunately means that clear conclusion and firm treatment protocol cannot be identified as of yet.
Of notable mention, a recently published cohort study of 42 patients evaluated the use of single-photon emission computed tomography (SPECT)/CT lymphoscintigraphy and SNB in early $\mathrm{T} 1 / 2$ papillary thyroid carcinomas in $\mathrm{cN} 0$ patients. ${ }^{27}$ The method which echoed methods now used in oral cancer gleaned very promising results, demonstrating that lymph node metastasis occurred in $46 \%$, of which $18 \%$ were located in the lateral neck compartments and that central node dissection could be avoided in $44.6 \%$. A true FNR could not be obtained, as routine central neck dissection was not performed on all patients; however, local compartments where SN were identified were cleared and based on this, an FNR of $8.1 \%$ was identified. This study revealed that $37.8 \%$ of patients would have been understaged, if conventional methods alone were used to evaluate the neck compartments.

\section{LARYNGEAL CARCINOMA}

The treatment of laryngeal cancer is dependent on tumor size, with the emphasis upon preserving function. Small tumors (T1-T2) can be treated by RT, transoral laser microsurgery, or transoral robotic surgery. Traditionally, more advanced tumors (T2b-3) are offered chemoradiotherapy; however, certain cases may be suitable for functionsparing surgery, such as vertical partial laryngectomy. In node-positive disease, it is recommended ${ }^{28}$ that levels II to $\mathrm{V}$ should be treated on the involved side. Elective treatment of the N0 neck is recommend in T3 and T4 disease. Radiotherapy or surgery \pm postoperative RT is provided to at least lymph node levels II, III, and IV bilaterally.

The incidence of occult cervical metastasis laryngeal cancer is proportionally related to the $\mathrm{T}$ staging and in T3/T4, disease is reported as $21.4-78 \% .^{29-31}$ Furthermore, there is a known association of risk of occult metastasis to site of primary tumor with the highest incidence in supraglottic $(40 \%)$, followed by subglottic $(30 \%)$ and glottis carcinomas $(10 \%) .^{32}$

Although the reported range is wide, it is likely patients are undergoing unnecessary treatment with the additional burden of both sides of the neck being affected. Additionally, there is controversy over the management of paratracheal nodes which are found to harbor occult metastasis in $9-27 \%$ of cases, but do not form part of the routine neck dissection. ${ }^{33}$

Several studies have evaluated the use of SNB in laryngeal carcinoma. ${ }^{32,34-38}$ These publications have typically included small study populations (10-50 participants) of previously untreated patients focusing on one subsite, or included all laryngeal subsites and some with inclusion of oral data. Further differences between these studies included the method of primary tumor control and SN detection (Table 1). As a result of these studies, it has been 


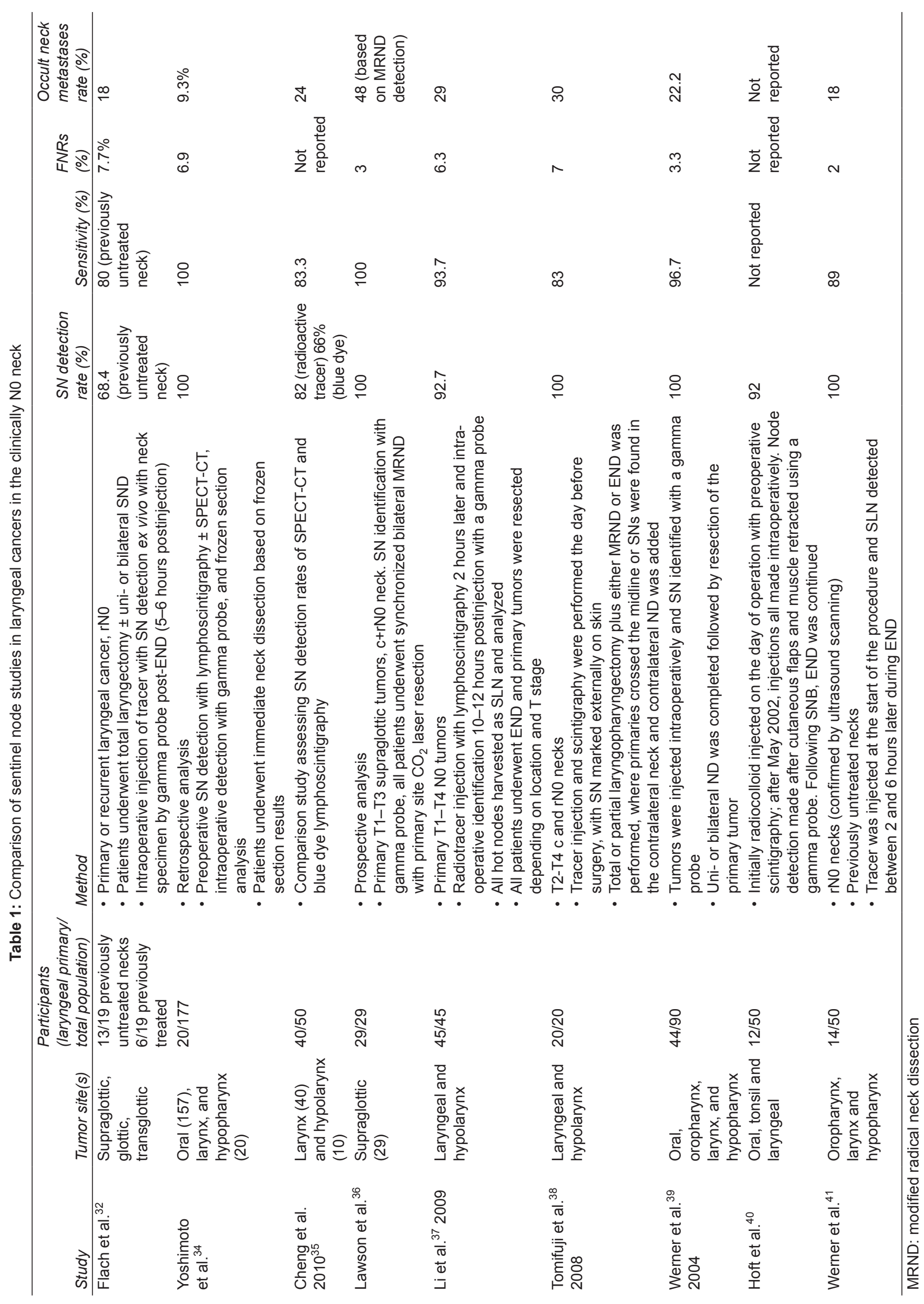


suggested that use of SNB may be better placed in patients undergoing transoral procedures rather than open surgery to avoid complications from a second surgery in the neck in the event of a positive SNB. ${ }^{32}$ Most of the studies reviewed used a reference of application of the $\mathrm{SN}$ concept in the clinically N0 patient and the majority of studies concluded that negative SN accurately concurred with pathological neck staging. However, one limitation for use of SLNB in laryngeal carcinoma patients may be the ability to inject radiotracer accurately around the margin of the tumor. Hoft et al. ${ }^{39}$ weakly established this in their study of 50 participants, in which four patients who underwent incomplete peritumoral tracer injection were excluded following the finding that all nodes accumulating tracer were identified as negative for disease with two patients (50\%) being found to harbor occult neck disease. Thus, it can be deduced that in patients where the caudal margin cannot be accessed for tracer application, the FNR of SNs biopsied may be increased and, as such, these patients may not be eligible for the staging technique. Studies since have sought to improve access to this margin and have developed application methods of use of winged butterfly cannulas. ${ }^{32}$ Caution is recommended in cases of difficult tumor visualization, where elective neck dissection may be indicated. In cases of more advanced laryngeal tumor staging (T3-T4), more evidence is required to show the advantage of a staged SN procedure over a one-stage primary tumor resection and elective neck dissection.

\section{SUMMARY}

The SNB has sound physiological basis that applies to solid tumors that spread via the lymphatic route. The technique has been described by many as in its infancy in tumors of the head and neck, but has shown great potential to answer some of the management-related controversies as outlined earlier. The promise of reducing treatment-related morbidity through harnessing technology to provide individualized treatment embodies the ethos of 21st-century medicine in the developed world. However, there are a number of potential areas where the SNB technique can be improved. Presently in oral cancer, the FNR of SNB is $14 \%$. In contrast, the equivalent failure rate of elective neck dissection is reported between $6 \%$ and $18 \%,{ }^{40-44}$ with most papers reporting neck recurrence after END in the pN0 neck to be $\leq 12 \% .{ }^{45}$ On balance, a slightly higher failure rate may be accepted for SNB in return for the improved morbidity and treatment cost, but ideally, it should be explored if it is possible to reduce the FNR to a level that is the same or better than the alternative treatment.

In terms of application to other solid head and neck cancers, there is more varied evidence. Certainly in the case of salivary gland tumors, isolated cases and small series have demonstrated that SLNB is a feasible technique and may even allow for a more conservative surgical approach to this heterogeneous group of tumors. Currently, however, there is a vast deficit in evidence supporting its use as a diagnostic tool in staging for these tumors and further large population trials are required to evaluate its potential value.

The use of SNB in thyroid and laryngeal carcinomas has been more widely evaluated and it has been well demonstrated that the use of SNB as a diagnostic tool can lead to more accurate staging of patients. It is of note, however, that larger, multicenter studies similar to the SENT trial have yet to be published for thyroid and laryngeal cancers and moving forward, it is what is required in order to wholly evaluate the use of a standardized technique in these patient groups and how results may impact and change the current conventional methods of surgical treatment.

\section{REFERENCES}

1. Essig H, Warraich R, Zulfiqar G, Rana M, Eckardt AM, Gellrich NC, Rana M. Assessment of cervical lymph node metastasis for therapeutic decision-making in squamous cell carcinoma of buccal mucosa: a prospective clinical analysis. World J Surg Oncol 2012 Nov;10:253.

2. Thompson CF, St John MA, Lawson G, Grogan T, Elashoff D, Mendelsohn AH. Diagnostic value of sentinel lymph node biopsy in head and neck cancer: a meta-analysis. Eur Arch Otorhinolaryngol 2013 Jul;270(7):2115-2122.

3. Morton DL, Wen DR, Wong JH, Economou JS, Cagle LA, Storm FK, et al. Technical details of intraoperative lymphatic mapping for early stage melanoma. Arch Surg 1992 Apr;127(4):392-399.

4. Schilling C, Stoeckli SJ, Haerle SK, Broglie MA, Huber GF, Sorensen JA, et al.. Sentinel European Node Trial (SENT): 3-year results of sentinel node biopsy in oral cancer. Eur J Cancer 2015 Dec;51(18):2777-2784.

5. Green B, Blythe J, Brennan PA. Sentinel lymph node biopsy for head and neck mucosal cancers-an update on the current evidence. Oral Dis 2016 Sep;22(6):498-502.

6. Sharma D, Koshy G, Grover S, Sharma B. Sentinel Lymph Node Biopsy: a new approach in the management of head and neck cancers. Sultan Qaboos Univ Med J 2017 Feb;17(1): e3-e10.

7. van der Vorst JR, Schaafsma BE, Verbeek FP, Keereweer S, Jansen JC, et al.. Near-infrared fluorescence sentinel lymph node mapping of the oral cavity in head and neck cancer patients. Oral Oncol 2013 Jan;49(1):15-19.

8. Alkureishi LW, Burak Z, Alvarez JA, Ballinger J, Bilde A, Britten $\mathrm{AJ}$, et al. Joint practice guidelines for radionuclide lymphoscintigraphy for sentinel node localization in oral/oropharyngeal squamous cell carcinoma. Ann Surg Oncol 2009 Nov;16(11): 3190-3210.

9. NICE Guideline (NG 36). Cancer of the upper aerodigestive tract: assessment and management in people aged 16 and over. London: NICE; 2016. [cited 2016 Feb 23]. Available from: http://www.nice.org.uk/guidance/ng36. 
10. Sood S, McGurk M, Vaz F. Management of salivary gland tumours: United Kingdom National Multidisciplinary Guidelines. J Laryngol Otol 2016 May;130(Suppl 2):S142-S149.

11. Stenner M, Molls C, Luers JC, Beutner D, Klussmann JP, Huettenbrink KB. Occurrence of lymph node metastasis in early-stage parotid gland cancer. Eur Arch Otorhinolaryngol 2012 Feb;269(2):643-648.

12. Wang X, Luo Y, Li M, Yan H, Sun M, Fan T. Management of salivary gland carcinomas-a review. Oncotarget 2017 Jan;8(3):3946-3956.

13. Armstrong JG, Harrison LB, Thaler HT, Friedlander-Klar H, Fass DE, Zelefsky MJ, et al. The indications for elective treatment of the neck in cancer of the major salivary glands. Cancer 1992 Feb;69(3):615-619.

14. Gould EA, Winship T, Philbin PH, Kerr HH. Observations on a "sentinel node" in cancer of the parotid. Cancer 1960 Jan-Feb;13:77-78.

15. Starek I, Koranda P, Zbooil V, Mrzena L. Sentinel lymph node biopsy in parotid gland carcinoma. Clin Nucl Med 2006 Apr;31(4):203-204.

16. Schilling C, Gnanasegaran G, McGurk M. Three-dimensional imaging and navigated sentinel node biopsy for primary parotid malignancy: new application in parotid cancer management. Head Neck 2014 Sep;36(9):E91-E93.

17. Moreno M, Moreno A, Tome C. Sentinel lymph node biopsy in adenocarcinoma of minor salivary gland. Oral Oncol 2008 Mar;44(3):305-308.

18. Teixeira G, Teixeira T, Gubert F, Chikota H, Tufano R. The incidence of central neck micrometastatic disease in patients with papillary thyroid cancer staged preoperatively and intraoperatively as N0. Surgery 2011 Dec;150(6):1161-1167.

19. Henry JF, Gramatica L, Denizot A, Kvachenyuk A, Puccini M, Defechereux T. Morbidity of prophylactic lymph node dissection in the central neck area in patients with papillary thyroid carcinoma. Langenbecks Arch Surg 1998 Apr;383(2): 167-169.

20. Zetoune T, Keutgen X, Buitrago D, Aldailami H, Shao H, Mazumdar M, et al. Prophylactic central neck dissection and local recurrence in papillary thyroid cancer: a meta-analysis. Ann Surg Oncol 2010 Dec;17(12):3287-3293.

21. Mallick U, Harmer C, Hackshaw A, Moss L; IoN Trial Management Group. Iodine or Not (IoN) for low-risk differentiated thyroid cancer: the next UK National Cancer Research Network randomised trial following HiLo. Clin Oncol (R Coll Radiol) 2012 Apr;24(3):159-161.

22. Raijmakers PG, Paul MA, Lips P. Sentinel node detection in patients with thyroid carcinoma: a meta-analysis. World J Surg 2008 Sep;32(9):1961-1967.

23. Balasubramanian SP, Harrison BJ. Systematic review and meta-analysis of sentinel node biopsy in thyroid cancer. $\mathrm{Br}$ J Surg 2011 Mar;98(3):334-344.

24. Falvo L, Marzullo A, Palermo S, Biancafarina A, De Stefano M, Vanni B, et al. The sentinel lymph node in papillary cancer of the thyroid including histological subtype. Ann Ital Chir 2006 Jan;77(1):13-18.

25. Arch-Ferrer J, Velazquez D, Fajardo R, Gamboa-Dominguez A, Herrera MF. Accuracy of sentinel lymph node in papillary thyroid carcinoma. Surgery 2001 Dec;130(6):907-913.

26. RubelloD,PelizzoMR,Al-NahhasA, SalvatoriM, O'Doherty MJ, Giuliano AE, Gross MD, et al. The role of sentinel lymph node biopsy in patients with differentiated thyroid carcinoma. Eur J Surg Oncol 2006 Nov;32(9):917-921..
27. Cabrera RN, Chone CT, Zantut-Wittmann DE, Matos PS, Ferreira DM, Pereira PS, et al. The Role of SPECT/CT lymphoscintigraphy and radioguided sentinel lymph node biopsy in managing papillary thyroid cancer. JAMA Otolaryngol Head Neck Surg 2016 Sep;142(9):834-841.

28. Jones TM, De M, Foran B, Harrington K, Mortimore S. Laryngeal cancer: United Kingdom National Multidisciplinary guidelines. J Laryngol Otol 2016 May;130(Suppl 2):S75-S82.

29. Mnejja M, Hammami B, Bougacha L, Chakroun A, Charfeddine I, Khabir A, et al. Occult lymph node metastasis in laryngeal squamous cell carcinoma: therapeutic and prognostic impact. Eur Ann Otorhinolaryngol Head Neck Dis 2010 Nov;127(5):173-176.

30. Kligerman J, Olivatto LO, Lima RA, Freitas EQ, Soares JR, Dias $\mathrm{FL}$, et al. Elective neck dissection in the treatment of T3/T4 N0 squamous cell carcinoma of the larynx. Am J Surg 1995 Nov;170(5):436-439.

31. Dias FL, Lima RA, Manfro G, Barbosa MM, Salviano S, Rocha RM, et al. Management of the N0 neck in moderately advanced squamous carcinoma of the larynx. Otolaryngol Head Neck Surg 2009 Jul;141(1):59-65.

32. Flach GB, Bloemena E, van Schie A, Hoekstra OS, van Weert S, Leemans $C R$, et al. Sentinel node identification in laryngeal cancer: Feasible in primary cancer with previously untreated neck. Oral Oncol 2013 Feb;49(2):165-168.

33. de Bree R, Leemans CR, Silver CE, Robbins KT, Rodrigo JP, Rinaldo A, et al. Paratracheal lymph node dissection in cancer of the larynx, hypopharynx, and cervical esophagus: the need for guidelines. Head Neck 2011 Jun;33(6):912-916.

34. YoshimotoS,Hasegawa Y,Matsuzuka T,ShiotaniA, TakahashiK, Kohno N, et al. Sentinel node biopsy for oral and laryngopharyngeal squamous cell carcinoma: a retrospective study of 177 patients in Japan. Auris Nasus Larynx 2012 Feb; 39(1):65-70.

35. Cheng Y, Wang BQ, Li SJ, Xia LJ, Li XF, Zhao DS. A comparative study of sentinel node detection in laryngeal and hypolaryngeal carcinoma by lymphoscintigraphy method and blue dye. Chin J Otorhinolaryngol Head Neck Surg, 2010,45(01): 42-46

36. Lawson G, Matar N, Nollevaux MC, Jamart J, Krug B, Delos M, et al. Reliability of sentinel node technique in the treatment of N0 supraglottic laryngeal cancer. Laryngoscope 2010 Nov;120(11):2213-2217.

37. Li ZW, Hu GH, Lei CM, Zhong SX, Li B, Hong SL, et al. Localization of sentinel lymph node with radionuclide in clinically N0 laryngeal and hypolaryngeal cancers. Chin J Otorhinolaryngol Head Neck Surg, 2009,44(05): 395-399.

38. Tomifuji M, Shiotani A, Fujii H, Araki K, Saito K, Inagaki K, et al. Sentinel node concept in clinically N0 Laryngeal and Hypolaryngeal cancer. Ann Surg Oncol 2008, 15(9); 2568-2575

39. Werner JA, Dunn AA, Ramaswamy A, Dalchow, Behr T, Moll $\mathrm{R}$, et al. The sentinel node concept in head and neck cancer: solution for the controversies in the N0 neck? Head Neck. 2004 Jul;26(7):603-611.

40. Ganly I, Goldstein D, Carlson DL, Patel SG, O'Sullivan B, Lee $\mathrm{N}$, et al. Long-term regional control and survival in patients with "low-risk," early stage oral tongue cancer managed by partial glossectomy and neck dissection without postoperative radiation: the importance of tumor thickness. Cancer 2013 Mar;119(6):1168-1176.

41. D'CruzAK, VaishR,KapreN,Dandekar M, GuptaS,Hawaldar R, et al. Elective versus therapeutic neck dissection in nodenegative oral cancer. N Engl J Med 2015 Aug;373(6):521-529. 
42. Yuen AP, Wei WI, Wong YM, Tang KC. Elective neck dissection versus observation in the treatment of early oral tongue carcinoma. Head Neck 1997 Oct;19(7):583-588.

43. Vandenbrouck C, Sancho-Garnier H, Chassagne D, Saravane D, Cachin Y, Micheau C. Elective versus therapeutic radical neck dissection in epidermoid carcinoma of the oral cavity: results of a randomized clinical trial. Cancer 1980 Jul;46(2): 386-390.
44. Yuen AP, Ho CM, Chow TL, Tang LC, Cheung WY, Ng RW, et al. Prospective randomized study of selective neck dissection versus observation for N0 neck of early tongue carcinoma. Head Neck 2009 Jun;31(6):765-772.

45. Ren ZH, Xu JL, Li B, Fan TF, Ji T, Zhang CP. Elective versus therapeutic neck dissection in node-negative oral cancer: Evidence from five randomized controlled trials. Oral Oncol 2015 Nov;51(11):976-981. 